\title{
Barriers to Providing High-Quality Nursing Care in Intensive Care Units: A Qualitative Study
}

\author{
Nasrin Rezaee ${ }^{1}$, Mahnaz Ghaljeh (iD) ${ }^{1}$ and Alireza Salar ${ }^{1, *}$ \\ ${ }^{1}$ Community Nursing Research Center, Zahedan University of Medical Sciences, Zahedan, Iran \\ "Corresponding author: Community Nursing Research Center, Zahedan University of Medical Sciences, Zahedan, Iran. Email: salar293@gmail.com
}

Received 2020 October 12; Accepted 2020 October 12.

\begin{abstract}
Background: Providing high-quality nursing care is the vision of healthcare systems. Several factors contribute to providing highquality care, which many of them need further investigation. In this line, qualitative research that helps researchers to access the thoughts and feelings of participants can play an important role in identifying care challenges.

Objectives: Therefore, this study aimed to identify nurses' concerns about the nursing care quality process in Intensive Care Units (ICUs).

Methods: In this study, a conventional content analysis was used for data analyses. Data were collected by unstructured interviews with 10 ICU nurses. Participants were selected using the purposive sampling technique. The sampling continued upon reaching data saturation.

Results: In total 290 codes were extracted, that using analysis and compare were categorized into three 3 main categories: "care barriers ", "motivational barriers ", and "management barriers ". Twelve sub categories were also extracted, including: "high workload", "not considering the educational needs", "not considering the work standards", "out of duty cares", "ward's bad structure", "personal motivation shortage", "poor work motivation", "personal and organizational motivation interference", "rules without work support", "work inconsistency in the ward", "keeping the position", and "inefficient communications between nurses and physicians".

Conclusions: In this study, barriers to nursing high-quality care and its related motivational and managerial dimensions were investigated. In other words, the present study identified barriers in different dimensions, and by identifying the effective factors in providing quality care has facilitated the implementation of measures to address the problems.
\end{abstract}

Keywords: Quality of Care, Intensive Care Unit, Nurses

\section{Background}

High-quality care is necessary to assure the patient's well-being and is the vision of nursing care services. Several studies reported that high-quality nursing care is associated with reduced hospitalization. Hence it's one of the main visions of healthcare systems. Hence, evaluating the quality of nursing care is a major part of hospital accreditation (1). Despite several years of research on concepts of nursing care quality, they are not well-understood. Quality of care also uses as a measure of patients' satisfaction (2). In a study conducted by Dabirian and colleagues on 70 patients infected with HIV/AIDS in Tehran, $72.9 \%$ of patients reported the quality of nursing care as moderate, $14.3 \%$ as poor, $11.4 \%$ as good, and $1.4 \%$ as very good (3). Zare and colleagues also reported that only $8 \%$ of nurses who participated in their study had a good performance (4). The literature review of the current study also revealed the poor quality of healthcare services in Iran (5). A survey of 12 studies conducted in European countries and the United States reported that the quality of nursing care had a poor level, and in some countries, patients were not satisfied with the care, such as Ireland and Greece (6).

Standards are the very foundation of improving the quality of care, improving the patients' outcomes, and reducing healthcare costs (7). Therefore, it seems necessary to recognize these standards and compare the nurse's performance against these standards. Nurses have a vital contribution in managing ICUs. Working in ICU requires a fresh mind and up-do-dated knowledge. Therefore, special attention should be paid to the clinical skills and knowledge of nurses working in ICUs (8). ICU hosts the patients with the most severe diseases in the hospital. Since ICU patients have a wide spectrum of diseases and often need highly specialized care, the incidence of nursing errors 
is high in ICU. On the other hand, in most cases, ICU patients are not capable of protecting themselves, for example, because of unconsciousness or being intubated (9). Besides, in ICU, nurses are straightly involved in patient's treatment, therefore, their knowledge and skills should be higher than their counterparts in other wards. This is possible if the nurse has the clinical skills and knowledge to provide care. Since patients with acute respiratory failure (ARDS) who are hospitalized at ICUs have better prognosis and survival, there is an increased demand for hospitalizing ARDS patients at ICUs. The important point is their longer hospitalization period. Therefore a special care plan is needed (10).

Although several recently conducted qualitative studies have investigated the quality of care from the perspective of patients and nurses, there are still controversies concerning various perceptions of the quality of care in different areas such as care activities, conflict response, and safe functioning. While based on the nurses' perspective, the quality of care is "good", patients have reported that the provided care couldn't meet their expectations $(11,12)$.

These differences can be attributed to several factors such as the ward that the patient was hospitalized, and people's beliefs and rules. One way to understand the challenges of providing quality care is to conduct qualitative research.

\section{Objectives}

Therefore, the present study was conducted to identify barriers to providing quality care in ICUs.

\section{Methods}

In this study, a conventional content analysis was used for data analyses. Content analysis is an important technique used in social science studies for deep analysis of data, particularly in nursing researches (13). Participants of this study were 10 nurses working in ICUs of hospitals affiliated to the Zahedan University of Medical Sciences in the City of Zaheda from 2015-16. Participants were selected using the purposive sampling technique. The sampling continued upon reaching data saturation. The inclusion criteria were having at least one year of working experience in ICUs and verbal consent to participate in the study. Data were collected by face-to-face semi-structured interviews. The interviews were started with the following question: "what nursing care do you provide to patients during a working shift?" to collect more information, the following questions were asked: "what do you mean about this" and "would you explain more about". The interviews lasted for
30-45 minutes. Interviews were recorded in MP3 formats, with the respondents' permission. Before performing interviews, the interviewees were informed about the objectives of the study. In total 10 interviews were conducted. Simultaneous with performing interviews, they were transcribed and further analyzed. The extracted codes were reviewed for categorization into themes. Several methods were used to increase the accuracy of the data. The extracted codes were provided to interviewees to obtain their opinions. The interviewees were assured about the confidentiality of the information. Participants could leave the study whenever they wanted. This study has been registered in Zahedan University of Medical Sciences (ethics code: IR.ZAUMS.REC.1394.426).

\section{Results}

In total 290 codes were extracted using the deep and rich description of participants' answers. After several times of review and summarizing the codes, they were categorized into 3 main categories: "care barriers ", "motivational barriers ", and "management barriers ". Twelve sub categories were named conceptually and abstractly (Table 1).

\subsection{Barriers Related to Care}

Participants clearly identified barriers to care in interviews. Based on this theme, due to the high number of patients and inadequacy of staff, they do not have enough time to perform all of their tasks, which maybe punished. The other sub-themes included "high workload", "not considering the educational needs", "not considering the work standards", "out of duty cares," and "bad structure of the ward". The participants noted that based on their experience, ICU has a high workload. In this line, an interviewee noted that: "usually in ICU each nurse has two patients, but sometimes the situation is different. In some shifts, we have three patients or two full patients and one common patient, which causes high workload" (Nurse No. 1).

Another participant noted that: "Nurses help the cleaning staff in changing the patient's position, which should be done once a day. Although patients need more repositions during a day, but because of the high number of patients and inadequacy of staff, this issue is not always following. Sometimes we do the reposition ourselves, because the nurse aides and cleaning services staff are not available" (Nurse No. 4).

About the secondary theme ("not considering the educational needs") an interviewee noted that: "When I was hired as an ICU nurse, they didn't provide me any training. 


\begin{tabular}{|c|c|}
\hline Sub Categories & Main Categories \\
\hline High workload & \multirow{3}{*}{ Care barriers } \\
\hline Not considering the educational needs, not considering the work standards & \\
\hline Out of duty cares & \\
\hline Personal motivation shortage & \multirow{3}{*}{ Motivational barriers } \\
\hline Poor work motivation & \\
\hline Personal and organizational motivation interference & \\
\hline Rules without work support & \multirow{4}{*}{ Management barriers } \\
\hline Work inconsistency in ward & \\
\hline Keeping the position & \\
\hline Inefficient communications between nurses and physicians & \\
\hline
\end{tabular}

I've learned everything here experimentally. It was really stressful to work in the ward. I didn't have enough knowledge. However, this is not just about me. No one here is trained for working in ICU." (Nurse No. 2)

Regarding the "not considering the work standards," an interviewee noted that: "According to the standards, each patient must be monitored by one nurse, but due to inadequacy of human resources, currently each nurse monitors three patients. Hence, due to the high workload, the provided care may not be standard. Meanwhile, administrators do not pay attention to this issue." (Nurse No 5.)

About the "out of duty care", a participant said: "We are doing tasks which are not officially related to us. I feel good when I did my patient's gavage correctly when the patient was delivered to the next shift, which means that I was able to do my patient's main care that day.. This means that I could provide good care on that day. We spend most of our time on tasks that are not related to us." (Nurse No. 6) Another participant said: "people's attitude would not change till they become familiar with nursing tasks. In fact, ICU nursing care is not considering an important issue. This upsets the nurse because their nursing care is marginalized". (Nurse No. 2)

Concerning the "bad structure of the ward" a participant noted: "our main problems are the beds and air mattresses, which are out of order and cause bedsores. In this ward with 16 patients, we have just two portable suctions, and ambo bags are few. For example, in cases with low levels of oxygen, we don't know what to do and only provide ambo. This problem relates to both the lack of equipment and structural defects of the building." (Nurse No. 5)

\subsection{Motivational Barriers}

Participants clearly mentioned several motivational obstacles. This theme contains the following sub-themes: "personal motivation shortage", "poor work motivation", and "personal and organizational motivation interference". Concerning personal motivation shortage, an interviewee noted: "heavy working shifts and high work pressure caused physical amortization in us. I have back pain and leg pain. I have more physical disorders than my colleagues who are working in other wards. I don't like to work in this ward anymore, and I am really disappointed." (Nurse No. 2)

Another participant said: "When it comes to evaluating ICU nurses' performance, we shouldn't judge them as not having enough experience or not being well-trained, but they are just too busy. Because of the high workload, the provided care may not be of high quality. In such situations, nurses don't have enough incentive to their best." (Nurse No. 7). Concerning the "poor work motivation," a participant noted: "when a physician does not pay attention to the nurse, it causes disappointment. The doctor's disregard for the nurse's opinions discourages the nurse, so we do not interact with them. Not paying attention to the nurse's opinion could make the nurse apathetic, which may negatively affect the patient's recovery." (Nurse No. 5)

Another participant noted: "our nursing system should be supported by matrons, supervisors, and the authorities in the nursing office, but unfortunately they rebuke nurses more than others. There are cases that high officials forgive the patient for his/her mistake, but the nursing staff act differently. These factors would kill the motivation of those working in the ward." (Nurse No. 8)

About the "personal and organizational motivation interference" a participant noted: "Human is error. We all 
make mistakes. Mistakes may be due to personal problems or life difficulties, or due to the working conditions. Nevertheless, these two issues may strength each other." (Nurse No. 3)

Another participant said: "Separating the work and personal life is inevitable, so that negative tasks at the workplace increase my negative energy and vice versa. When I'm calm, I will do my job well. But most of the time, I have negative energy in the ward and a simple spark in the ward would lead to my explosion!" (Nurse No. 10)

\subsection{Managerial Barriers}

This theme comprised of the following sub-themes: "rules without work support", "inconsistency of tasks", "keeping the position", and "inefficient communication between nurses and physicians". Regarding the "rules without work support", an interviewee noted: "every day they make a new rule. For example, they want to prevent medical errors and conduct a system like industrial countries. When the ICU is not well-equipped, human resources are not sufficient, beds are insufficient, and medical knowledge is not enough, why do we make terrible rules. With these rules, we just store unnecessary information which have no value, instead of providing care to patients." (Nurse No. 10)

Regarding the "inconsistency of tasks", an interviewee noted: "physicians come so late for visiting patients, which is usually at the end of the shift change and they order time-consuming tasks such as CT and MRI that you had to do them because the radiology ward only performs emergency CT scans at evening shifts. The poor nurse should beg two persons here first the physician to come and do the visit earlier and then the radiologist to do the job for God's sake. These issues cause inconsistency in the workplace." (Nurse No.5). Another participant said: "although they held so many meetings but the responsible physicians is not clear. In the ICU, it is not clear whether the anesthesiologist is the first to speak or the patient's physician. This confuses the nurse, and I am confused as the nurse here, and I don't know with whom I should share the patient's information. Finally, I say to both of them, and each of them gives a separate order! Because of this, I call so many times to clear the matter for the patient. This wastes my time and energy and I won't have enough time to do my main tasks." (Nurse No. 7)

Regarding the "keeping the position," a participant said: "nursing managers are completely obeying the orders of higher authorities and physicians. They don't think about anything and just obey the orders and defend the decisions of higher authorities. It seems that they don't know the declaration. Even when they are not satisfied with a rule, they say nothing to keep their position. They are like hammers of authorities and physicians on the nursing system's head. Nursing managers just use the punishment of doctors against us, instead of supporting us." (Nurse No. 9)

Concerning the "inefficient communication between nurses and physicians", a participant said: "the communication system between nurses and doctors and other groups is full of problems. Doctors have problems with each other and can't directly talk to each other, which causes severe problems for the nursing staff. Anesthesiologists and physicians challenge each other, and the patient and the nurse are not important, and this affects patient care." (Nurse No. 7)

\section{Discussion}

The present study aimed to identify barriers to provide high-quality nursing care in ICU. The obtained codes were categorized into three main categories, as follows: "Barriers to provide care", "motivational barriers", and "managerial barriers". The findings indicated these factors influence the quality of care in ICU.

"Barriers to providing care" were identified as the main category with the following sub-themes: high workload, not considering the educational needs, not considering the work standards, out of duty cares, and bad structure of the ward. There are other studies which showed that barriers to care play an important role in providing high-quality care. This finding is consistent with the study of Nobahar et al. (2014) which showed that the structure of the ward and its facilities affect the quality of care (14). There are quantitative studies that showed that the equipment of the ward plays an important role in the quality of patient care. A study conducted in the ICUs of the Mazandaran province reported that ICU nurses had a high workload (15). A review study reported that ICU nurses in various countries experience different workloads (6). In this regard, Om Hashem et al. (2013) argued that to maintain ICU nurses their workload should be reduced (16). Fallahian et al. (2013) showed that the ICU was not built according to the standards and suggested that improving structural standards guarantees the quality and optimal performance in the ward (17). The observed difference in the workload of ICU nurses can be attributed to the number of staff, patients' profile, and severity of diseases as well as laws and regulations governing the provision of medical services. In line with the lack of attention to educational needs, Alaei et al. (2012) showed that training the staff of ICU by their clinical supervisors positively affects the performance of nurses (10). Sadeghian et al. (2017), in a study on improving the quality of care in hemodialysis wards, showed that in addition 
to the knowledge and skills of nurses, they should also be trained in the emotional dimension, which helps the nurses to provide high-quality care (18). Ahmadi et al. (2011) reported that the availability of resources is a sufficient and appropriate feature of special wards. Therefore, lack of equipment or their breakdown will reduce the quality of care. Lack of equipment and facilities increases the workload of nurses and negatively affects them, which causes waste of time and energy that, in turn, leads to fatigue and lower performance. Therefore, the sufficiency of resources can reduce the work problems of nurses and improve the quality of nursing services (19). In the present study, one of the reasons for the low quality of care was doing tasks that are not related to the nursing staff, which causes increased work pressure. There are arguments about the negative association between the number of staff and the workload of the staff, which in turn affects the quality of the provided car (20). It seems in cases with a high number of patients and a low number of nursing staff, the staff may focus only on the main tasks, instead of tasks that should be done by others. It worth noting that in some texts, ineffective care has been referred to as pointless care (21). In fact, it can be inferred that barriers to care lead to poor care.

Motivational barriers were another category of the present study with the following sub-themes: "lack of individual motivation", "lack of organizational motivation", and "interference of individual and organizational motivation". In a qualitative study that investigated nurses' motivational factors, individual and organizational factors such as encouragement, interpersonal respect, meeting expectations, and consultation were identified as influential and promoting motivational factors in how nurses work together (22). As the findings of several quantitative and qualitative studies show, motivation influences how individuals perform their tasks and work with other nurses; Therefore, if individual and intra-organizational motivational factors are not considered, they can be considered as a barrier to good performance and, of course, the quality of optimal care.

Managerial barriers was another extracted them with the following sub-themes: "Unsupported Rules", "Welfare Disorder", "Maintaining Position", and "Dysfunctional Physician-Nurse Communication". Rules without work support, work inconsistency in the ward, keeping the position, and inefficient communications between nurses and physicians. In a quantitative study, managerial barriers reported by nurses included the shortage of human resources, lack of financial resources, and inadequacy of equipment (23). Another qualitative study mentioned the behavior of nursing managers as well as some characteristics of nursing managers, including lack of support from staff and disregard for nurses' opinions, dictatorship and obedience, trying to maintain position, discrimination, and insignificance, as the barriers to productivity. Organizing and guiding health staff were among the most important managerial barriers (24). Some of these barriers, such as maintaining the position, are also found in the present study. In the sub-category of dysfunctional communication between physician and nurse, which was in line with the study of Shukri et al. (2013) in which the study of nurses reported that their professional relationship with physicians is moderate and the most important factor in disrupting the professional relationship The nurse did not seek medical advice from the patient-nurse in performing medical procedures, while in the same study, the physicians believed that they had a good relationship with the nurses (25). A qualitative study on nurses' professional communication experiences has investigated such communications in the context of physician-patient communication deficit and reported that nurses fill the gap between the doctor and the patient (26).

In this regard, a qualitative study on the quality of care provided by ICU nurses reported that "adequate and efficient human resources", "human communication", "organizing the performance of the nurse", and "optimal environment, tools, equipment, and appropriate facilities are essential for providing high-quality care (14). Also, a study aimed to determine factors that affect the communication between nursing managers and nurses, from the perspective of nurses, reported that human, organizational, and communication-related factors are effective in explaining the communication between nursing managers and nurses. Factors that influence the relationship between nursing managers and nursing staff play a role in improving the quality of nursing care (27).

\subsection{Limitations}

As observation plays a crucial role in collecting data for qualitative studies, not using this method in the present study can be considered as a limitation. The authors suggest performing further studies by other approaches with a special focus on observation.

\subsection{Conclusion}

This study described the barriers to providing highquality care from the perspective of ICU nurses. Based on the findings, to improve the quality of care, barriers related to providing care, motivations, and management should be addressed. The findings provided an overview of the barriers to quality care and showed that to provide quality care, all barriers should be considered, regardless of all aforementioned issues, improving quality care 
would be an important challenge. Finally, it can be argued that effective nursing management is an important step towards removing barriers to providing care, motivation, and management. Administrators of the nursing staff should change the management system and ultimately improve the quality of care using the findings of the present study.

\section{Acknowledgments}

This study is part of a larger research that is approved by the Zahedan University of Medical Sciences. The study is also approved by the ethics committee of the Zahedan University of Medical Sciences (code: 7734). The authors thank all officials and participants who helped us.

\section{Footnotes}

Authors' Contribution: Nasrin Rezai: data collection and manuscript composition and study design. Mahnaz Ghaljeh: study design and manuscript composition. Alireza Salar: study design and data analysis: data collection and study design.

Conflict of Interests: The authors declare no conflict of interest.

Ethical Approval: This research was approved by the Zahedan University of Medical Sciences, and all ethical considerations of clinical studies were observed by the authors.

Funding/Support: No financial support was received for this research.

Informed Consent: Written informed consent was obtained from all patients.

\section{References}

1. Zamanzadeh V, Valizadeh L, Tabrizi FJ, Behshid M, Lotfi M. Challenges associated with the implementation of the nursing process: A systematic review. Iran J Nurs Midwifery Res. 2015;20(4):411-9. doi: 10.4103/1735-9066.161002. [PubMed: 26257793]. [PubMed Central: PMC4525336].

2. Salehian M, Heydari A, Moonaghi HK, Aghebati N. Developing the concept of caring in nursing education. Electron Physician. 2017;9(5):4425-33. doi: 10.19082/4425. [PubMed: 28713517]. [PubMed Central: PMC5498710].

3. Dabirian A, Zolfaghari H, Saidi ZA, Alavi-Majd H. Views of AIDS patients regarding nursing care quality in healthcare centers affiliated to Shaheed Beheshti and Tehran Universities of Medical Sciences. Advances in Nursing \& Midwifery. 2008;17(61):39-45.

4. Zarea K, Negarandeh R, Dehghan-Nayeri N, Rezaei-Adaryani M. Nursing staff shortages and job satisfaction in Iran: issues and challenges. Nurs Health Sci. 2009;11(3):326-31. doi: 10.1111/j.1442-2018.2009.00466.x. [PubMed: 19689643].
5. Kasa AS, Gedamu H. Predictors of adult patient satisfaction with nursing care in public hospitals of Amhara region, Northwest Ethiopia. BMC Health Serv Res. 2019;19(1):52. doi: 10.1186/s12913-0193898-3. [PubMed: 30665400]. [PubMed Central: PMC6341709].

6. Aiken LH, Sermeus W, Van den Heede K, Sloane DM, Busse R, McKee $\mathrm{M}$, et al. Patient safety, satisfaction, and quality of hospital care: cross sectional surveys of nurses and patients in 12 countries in Europe and the United States. BMJ. 2012;344. e1717. doi:10.1136/bmj.e1717. [PubMed: 22434089]. [PubMed Central: PMC3308724].

7. Lotfi M, Zamanzadeh V, Valizadeh L, Khajehgoodari M, Ebrahimpour Rezaei M, Khalilzad MA. The implementation of the nursing process in lower-income countries: An integrative review. Nurs Open. 2020;7(1):42-57. doi: 10.1002/nop2.410. [PubMed: 31871690]. [PubMed Central: PMC6917928].

8. Yaghubi M, Sharifi SH, Abbaspour H. Knowledge, attitude, and practice of intensive care units nurses about nosocomial Infections control in hospitals of Bojnurd in 2012. Journal of North Khorasan University of Medical Sciences. 2014;5(5):943-50. doi: 10.29252/jnkums.5.5.S5.943.

9. Jiang SP, Chen J, Zhang XG, Lu XY, Zhao QW. Implementation of pharmacists' interventions and assessment of medication errors in an intensive care unit of a Chinese tertiary hospital. Ther Clin Risk Manag. 2014;10:861-6. doi: 10.2147/TCRM.S69585. [PubMed: 25328401]. [PubMed Central: PMC4199561].

10. Alaee V, Seyedjavadi M, Mohammadi R, Aslani MR, Hashemi-biria B. The effect of clinical supervisors education to quality of nursing care of patients under mechanical ventilation in critical care units. Journal of Health And Care. 2012;14(3):0.

11. khaki S, Esmaeilpourzanjani S, Mashouf S. Nursing cares quality in nurses. Scientific Journal of Nursing, Midwifery and Paramedical Faculty. 2018;3(4):1-14. doi:10.29252/sjnmp.3.4.1.

12. Sharafi S, Chamanzari H, Rajabpour M, Maghsoudi S, Mazloom SR. Comparative Study on the Effect of "Primary Nursing" and "Case Method” on Patients' Quality of Physical Care. Journal of Health Promotion Management. 2016;5(4):17-23.

13. Graneheim UH, Lundman B. Qualitative content analysis in nursing research: concepts, procedures and measures to achieve trustworthiness. Nurse Educ Today. 2004;24(2):105-12. doi: 10.1016/j.nedt.2003.10.001. [PubMed: 14769454].

14. Nobahar M. Care quality in critical cardiac units from nurses perspective: a content analysis. Journal of Qualitative Research in Health Sciences. 2014;3(2):149-61.

15. Alizadeh M, Heidari Gorji MA, Khalilian AR, Esmaeili R. Assessment of nursing workload and related factors in intensive care units using the nursing activities score. Journal of Mazandaran University of Medical Sciences. 2015;24(122):147-57.

16. Ragab OHG, Al Torky MAM, Abdalla SM. Nurses' Workload and its' Impact on Productivity in Trauma and Post Operative Intensive Care Units at Assiut University Hospital. Journal of American Science. 2013;9(12):927-39.

17. FALAHINIA GH, Zareian A, oshvandi K, farhanchi A, MOGHIMBEIGI ABBAS. Comparison of intensive care units Structural Standards. IJCCN. 2013.

18. Shahgholian N, Dashti Dehkordi AR, Sadeghian Z. PERSPECTIVE OF HEMODIALYSIS PATIENTS AND NURSES IN RELATION WITH CARING BEHAVIORS. The J Urmia Nurs Midwifery Fac. 2017;15(9):659-66.

19. Ahmadi F, Nobahar M, Alhani F, Falahi Khoshknab M. Perspectives of retired nurses on factors that affect quality of nursing care. Journal of hayat. 2011;17(1):24-34.

20. Schubert M, Ausserhofer D, Desmedt M, Schwendimann R, Lesaffre $\mathrm{E}, \mathrm{Li} \mathrm{B}$, et al. Levels and correlates of implicit rationing of nursing care in Swiss acute care hospitals-a cross sectional study. Int J Nurs Stud. 2013;50(2):230-9. doi: 10.1016/j.ijnurstu.2012.09.016. [PubMed: 23084600].

21. Aghabarari M, Dehghan Nayeri N. Futile Care: challenges of applying futility concept in caring domain. Journal of hayat. 2015;21(1):1-5. 
22. ZAMANZADEH V, IRAJPOUR AR, VALIZADEH L, SHOHANI M. MOTIVATIONAL FACTORS AFFECTING COLLABORATION AMONG NURSES: A QUALITATIVE STUDY. AVICENNA JOURNAL OF NURSING AND MIDWIFERY CARE. 2015.

23. Babaeipour-Divshali M, Amrollahi-Mishavan F, Firouzkouhi MR. Evaluation of scales and barriers of managerial performance of head nurses based on BARS performance evaluation model in Rasht, 2011. Journal of Clinical Nursing and Midwifery. 2015;4.

24. Dehghan Nayeri N, Nazari AA, Salsali M, Ahmadi F, Adib Hajbaghery M. Iranian staff nurses' views of their productivity and management factors improving and impeding it: a qualitative study. Nurs Health Sci. 2006;8(1):51-6. doi: 10.1111/j.1442-2018.2006.00254.x.
[PubMed: 16451429].

25. Shokri A, Yazdan Panah A, Vahdat S. The professional relationship between the nurses and physicians from their own point of view.Journal of Health and Care. 2013;15(1):76-69.

26. Azimi Lolaty H, Ashktorab T, Bagheri Nesami M, Bagherzadeh Ladari R. Experience of professional communication among nurses working in educational hospitals: a phenomenological study.Journal of Mazandaran university of medical sciences. 2011;21(85):108-25.

27. Farajzadeh Z, Karimi Z. Factors Affecting the Effective Relationship between Nursing Managers and Nurses from the Nurses' Viewpoint.JNE. $2020 ; 8(6)$. 